\title{
Mechanism of protein phosphatase-2Aregulating phosphorylation of amyloid precursor proteosome and $\mathrm{A} \beta$ generation
}

\author{
Zhang JW, Jing LJ, Jian G, Dong GC \\ Baotou Teachers' College, Inner Mongolia University of Science \& Technology, Baotou, Inner Mongolia, P.R China. \\ dongguicheng78@163.com
}

\begin{abstract}
Objective:To discuss whether PP-2A influences the phosphorylation level at APP threonine 668 locus thus regulating $A \beta$ secretion.

Method: In the experiment, 24 hours after N2a cells of stably transfected human APP (N2a/APP) were treated with okadaic acid (OA) or DES (C6-ceramide) (N2a/APP), an injection of OA cerebral stereotactic was administered to a SD rat in the hippocampal region, or PP-2A overexpressed plasmids was transfected transiently, the aggregate levels of APP phosphorylated APP, and APP-CTF were detected through immunoblotting and the activity of PP-2A and secretase was also detected using a reagent kit.

Result: The phosphorylation level of APP was significantly increased after the PP-2A activity was inhibited by OA. DES activated PP-2A or over-expressed PP-2A was able to reduce the phosphorylation level of APP. Either can inhibit PP and reduce the phosphorylation level of APP. The level of phosphorylated APP was increased significantly after the SD rat was injected with OA through the hippocampal region. The activity of $\beta$ - and $\gamma$-secretases in N2a/APP cells significantly increased after OA treatment whereas the $\alpha$-secretase activity had no significant changes; the $A \beta$ level increased.

Conclusion: We discovered that PP-2A was capable of regulating the A $\beta$ level by regulating APP phosphorylation level and $\beta$ and $y$-secretase activity(Fig. 5, Ref. 30). Text in PDF www.elis.sk.

Keywords:PP-2A,alzheimer disease, phosphorylation, $A \beta$, protein.
\end{abstract}

\section{Introduction}

Alzheimer's disease (AD) is a progressive neurodegenerative disease and its histopathological changes mainly include formation of a large number of extracellular senile plaques and entanglement of intracellular neurofibriles. The main component of the senile plaque is $\beta$-amyloid protein $(A \beta)$ and malnutrition-related neuritis is generated in its vicinity (1). $A \beta$ is generated in such a way that the amyloid precursor proteosomeis severed continuously by two protein secretases, e.g. $\beta$-secretase (BACE- 1$)$ and $\gamma$-secretase (presenilin, PS-1/PS-2) (2). In amyloid pathologic changes, products resulted from $\beta$-secretase severing, e.g. $\mathrm{N}$-terminal soluble segment (sAPP- $\beta$ ) and $\mathrm{C}$-terminal segment can continue to be severed by $\gamma$-secretase and produce $A \beta$ polypeptide finally (3). The toxic actions of $A \beta$ are extensive and capable of causing cell death. The mechanisms mainly include: peroxidation damage, influencing calcium balance, activating aspartase proteinase, stimulating protein phosphorylation, mitochondria abnormality etc. (4). In addition, A $\beta$ fibril can also specifically lead to neuron malnutrition. In the rat cortical neuron undergoing primary culture, over-expressed APP may lead to cell apoptosis, whereas $\gamma$-secretase inhibitor is

Baotou Teachers' College, Inner Mongolia University of Science \& Technology , Baotou, Inner Mongolia, P.R China

Address for correspondence: GC Dong, Baotou Teachers' College, Inner Mongolia University of Science\& Technology, No.3, Kexue Road, Qingshan District, Baotou, InnerMongolia, P.R China capable of blocking such apoptosis (5). In the transgenic rat model, $\mathrm{A} \beta$ aggregation can lead to malnutrition-related neuroinflammation, pathologic changes in tau protein, neuronal death, and DNA damage (6). When APP is over expressed or severed unusually, $\mathrm{A} \beta$ will form a toxic oligomer, deposit and form senile plaque, and cause damage to the learning memory (7). It has been reported that gene mutation of APP and PS-1 is the major cause of increased generated $\mathrm{A} \beta$ in the genetic $\mathrm{AD}$ disease. However, the cause of a significant rise of $\mathrm{A} \beta$ in the majority of sporadic $\mathrm{AD}$ patients still remains unclear (8).

In the research, we have discovered that overexpression or medication activated PP-2A in N2a/APP (stably transfected human APP695) cells could lower the phosphorylation level of 668 locus. The phosphorylation level of 668 locus could be lowered and generated A $\beta$ increased after the PP-2A activity was inhibited. Meanwhile, we have also discovered that PP-2A was able to influence the activity of APP-related secretases and PP-2A could regulate secretion of $\mathrm{A} \beta$ by influencing phosphorylation and secretase activity of the threonine 668 locus.

\section{Material and methods}

\section{Material}

DMEM, OPTI-MEM, foetal bovine serum (FBS), and neomycin (G418) were purchased from Beijing Ruixiang Biotechnology Co., Ltd. The BCA protein determination reagent kit was purchased from U.S. SantaCruz Company, and the plasmid midi 
preparation kit was purchased from U.S. Amresco Company. The tetramethylbenzidinecolor developing agent was purchased from Beijing Biocoen Biotechnology CO.,Ltd. The DNA molecular weight marker was purchased from Beijing Bole Bioscience Development Co. Ltd. The reverse transcription reagent kit and Taq enzyme were purchased from Beijing CoWin Bioscience Co., Ltd. The OA was purchased from U.S. Amresco Company. The Lipofectamine TM2000 transfection reagent was purchased from U.S. Amresco Company.

The cryogenic refrigerator was purchased from LEAD-TECH (Shanghai) Scientific Instrument Co., Ltd. The incubator-co2 was purchased from Zhengzhou Nanbei Instrument Equipment Co., Ltd. The inverted microscope was purchased from U.S. MEDICA Company. The gel electrophoresis analytic system was purchased from U.S. Labnet Company. The multi-function microplate reader was purchased from Beijing Jingli Centrifuge Co. Ltd.

\section{Treatment of animal samples}

The SD rats were purchased from Shanghai Laboratory AnimaI Centre of The Chinese Academy of Sciences, Conformity Certificate No. SCXK (Shanghai) 2013-0042 and all animals were in accordance with Law of the People's Republic of China on Inspection and Quarantine of Animals and Plants.

All animals were given sufficient water and food. The rats were sacrificed after intraperitoneal injection anaesthesia. The rats were decollated immediately after death and placed on the ice. The skull was scissored from the occipital bone hole of the centre of the head with scissors to remove the bones. The brain was raised along the edge of the cranial cavity with tweezers and placed on a piece of filter paper for blunt dissection of the hippocampus. The hippocampal part of the rat brain was taken out quickly, numbered as per EP tube, weighted respectively, and stored in a refrigerator at $-80^{\circ} \mathrm{C}$ for homogenate in the future (9).

It was boiled for 10 minutes after homogeneous mixing of the tissue block, centrifuged at a room temperature at $8000 \mathrm{~g}$ for 10 minutes. The insoluble components were taken out with a pinhead and the supernatant was stored in a refrigerator at $-80^{\circ} \mathrm{C}$ for use in subsequent experiments.

\section{Stereotactic injection to rat brain}

20 SD rats weighing 200-220g were divided into the two groups with 10 for each group. The SD rats were anesthetized by $0.3 \%$ chloral hydrate, fixed onto the brain stereotactic instrument, cut in the centre of the top of the head to expose the bregma after the fur was removed and skin was sterilized. A microsyringe was inserted vertically by $2.5 \mathrm{~mm}$ from the brain surface at $3.0 \mathrm{~mm}$ behind the bregma and $2.5 \mathrm{~mm}$ right of the centre line in accordance with the rat brain orthostatic map. The bilateral hippocampal regions were gently injected with $5 \mu \mathrm{l}$ for $3 \mathrm{~min}$. The syringe needle was retracted gently after retaining for 5 minutes. All operation procedures were performed under an aseptic condition. Penicillin was applied to the skin incision site for antibiosis and the wound was sutured. The rats of the sham-operated group were injected with normal saline of an equivalent volume.

\section{Cell culture and transfection administration}

Mouse neuroblastoma cells (N2a) were purchased from Shanghai Boyan Biotechnology Co., Ltd. N2a cells of stably transfected human APP (N2a/APP). The cells were cultured in the incubator containing $5 \% \mathrm{CO}_{2}$ at $37^{\circ} \mathrm{C}$ and the culture medium was replaced at an interval of 3 days. The cells could be used for passaging or further experiment when the density reached approximately $90 \%$.

LipofectAMINETM 2000 reagent was used for plasmid transfection in strict accordance with the instructions. The cells were transferred to a 96-well micro-plate and transfection was performed until the cell density reached $80-90 \%$. The culture medium containing no blood serum neither antibiotic was used, replaced after 6 hours, and corresponding cell treatment and detection within 48 hours.

\section{Extraction and concentration determination of protein samples}

The tissue sample was stored in a cryogenic refrigerator following homogenization. Partial homogenate was taken and $2 \times$ SDS sample buffer of an equivalent volume was added, PMSF, protease inhibitor, subject to lysis on ice for 30 minutes. The lysis solution may freeze and mixing was performed at an appropriate interval. Then it was water bathed for 10 minutes and subjected to $20000 \mathrm{~Hz}$ ultrasound 3s X15 times at the amplitude of approximately 20$30 \%$. Excessive violent operation should be avoided to prevent the histone bound DNA from being broken. It was centrifuged at $8000 \mathrm{~g}$ for 3 minutes. The supernatant was collected. The protein concentration was tested with the BCA method (10).

\section{Western blotting}

The separation gel was prepared in accordance with the molecular weight of the antigen. $3 \mathrm{ml}$ separation gel was infused into the gel trough and sealed after being full of double distilled water, $2 \mathrm{ml}$ spacer gel prepared was added to the gel trough after solidification. A broach was inserted and the electrophoresis channel was marked. The electrophoresis channel was washed with double distilled water and filled with electrophoretic buffer solution. Specimen of the identical quality was taken for polyacrylamide gel electrophoresis and $20 \mu \mathrm{g}, 30 \mu \mathrm{g}$ and $40 \mu \mathrm{g}$ specimen were added on the basis of contents of different antigens in the cells. $5 \%$ skim milk powder was diluted with TBS and shaken slowly in a shaking table for 1 hour or stored in a refrigerator at $4{ }^{\circ} \mathrm{C}$ overnight after addition of properly diluted primary antibody. It was washed with $1 \times$ TBS buffer solution, 10 min for each time. The Odyssey two colour fluorescence second antibody was incubated for $1 \mathrm{~h}$ after diluted as per 1:1000. It was washed with $1 \times \mathrm{TBS}$ buffer solution for 3 times, 10 minutes for each time; slightly washed with double distilled water and developed by Odyssey scanning.

\section{ELISA adsorption method}

G2-10 stock solution was diluted with $100 \mathrm{mM} \mathrm{NaHCO}_{3},(\mathrm{pH}$ 9.6), $1 \mu \mathrm{g} /$ well, G2-11 stock solution was diluted with $100 \mathrm{mMTris-}$ $\mathrm{Cl},(\mathrm{pH} 8.4), 1 \mu \mathrm{g} /$ well. The coated antibody was added to a high affinity 96 -well ELISA plate with $100 \mu \mathrm{l} /$ well. The ELISA plate was placed in a wet box at $4{ }^{\circ} \mathrm{C}$ overnight. It could be incubated for two nights if the final developing signal was excessively low 


\section{4-190}

during A $\beta 42$ detection. The coated antibody was thrown away. The wells were filled with TBS and rinsed on the shaking table for 3 times and 3 minutes. Each well of the ELISA plate was filled with TBS containing 5\% BSA, blocked at room temperature for 2 hours. The ELISA plate was rinsed with TBS (TTBS) containing $0.1 \%$ Tween-20 for 3 times and 3 minutes. 10\% BSA was diluted as per 1:100 and biotin-labelled 4G8, or diluted as per 1:500 and biotin-labelled WO2, $20 \mu \mathrm{l} /$ well. Then, $10 \mu \mathrm{l}(\mathrm{A} \beta 40)$ or $40 \mu \mathrm{l}(\mathrm{A} \beta 42)$ animal homogenate supernatant or cell culture media supernatant was added and normal saline or culture media was to supplement the insufficient part of the total volume of $100 \mu 1$ per well. It was stored at $4^{\circ} \mathrm{C}$ overnight. The sample and antibody were thrown away. The culture plate was rinsed with TTBS for 3 minutes. The avidinlabelled horse radish peroxidase HRP-NeutroAvidin $(1 \mathrm{mg} / \mathrm{ml})$ was diluted as 1:1000 with $2 \%$ BSA. $100 \mu$ lvidin-labelled horse radish peroxidase was added to each well and placed on the shaking table for incubation at room temperature for 1 hour. The culture plate was rinsed for 5 times with TTBS and once with TBS for 3 minutes after the HRP was thrown away. $100 \mu \mathrm{TMB}: \mathrm{H}_{2} \mathrm{O}_{2}(1: 1)$ solution was added to each well to observe changes in colour. $50 \mu \mathrm{l}$ of $1 \mathrm{M} \mathrm{H}_{2} \mathrm{SO}_{4}$ was added to each well immediately after the solution became blue. At the time, the solution colour would become yellow. The absorbance value was read at a wave length of $450 \mathrm{~nm}$ on the microplate reader and the content was calculated as per the standard curve.

\section{Stereotactic injection to rat brain}

20 SD rats weighing 200-220g were divided into two groups with 10 for each group. The SD rats were anesthetized by $0.3 \%$ chloral hydrate, fixed onto the brain stereotactic instrument, cut in the centre of the top of the head to expose the bregma after the fur was removed and skin was sterilized. A microsyringe was inserted vertically by $2.5 \mathrm{~mm}$ from the brain surface at $3.0 \mathrm{~mm}$ behind the bregma and $2.5 \mathrm{~mm}$ right of the center line in accordance with the rat brain orthostatic map. The bilateral hippocampal regions were gently injected with $5 \mu \mathrm{l}$ for $3 \mathrm{~min}$. The syringe needle was retracted gently after retaining for 5 minutes. All operation procedures were performed under an aseptic condition. Penicillin was applied to the skin incision site for antibiosis and the wound was sutured. The rats of the sham-operated group were injected with normal saline of an equivalent volume.

\section{Data statistics}

The gray values of the immunoblotting stripes were calculated with the Odyssey software for semiquantitative analysis. The immunofluorescence gray values were calculated with the Image-ProPlus 6.0. The data was expressed with the mean number \pm standard deviation (mean $\pm \mathrm{SD})$. The one-way analysis of variance or $\mathrm{T}$ test was used for comparison among groups. The statistical software, EXCEL, was used to analyse and process the data.

\section{Results}

\section{Inhibition of PP1 in reducing phosphorylation level of APP}

As a protein phosphatase inhibitor, OA can inhibit activity of PP-2A and PP1 respectively within different dosage ranges. The inhibition ranges are: $0.1-10 \mathrm{nM}$ specificity inhibition PP-2A. The OA above $10 \mathrm{nM}$ is able to inhibit the activity of both PP-2A and PP1. As a threonine/serine esterase, PP1 may also influence the

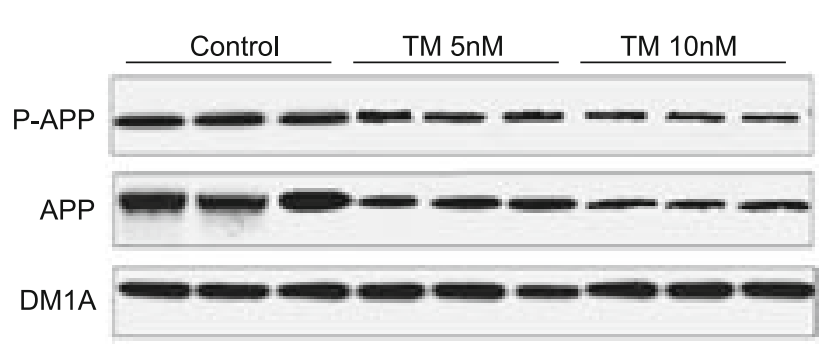

(A)

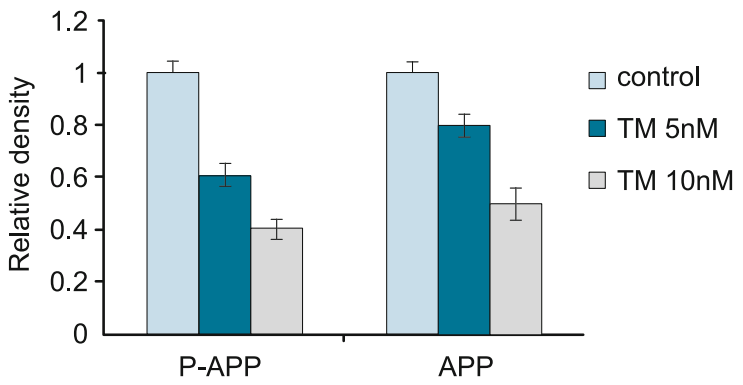

(B)

Fig. 1. Inhibit PP1 by Decreasing PAPP Activity in N2a/APP Cells via TM.
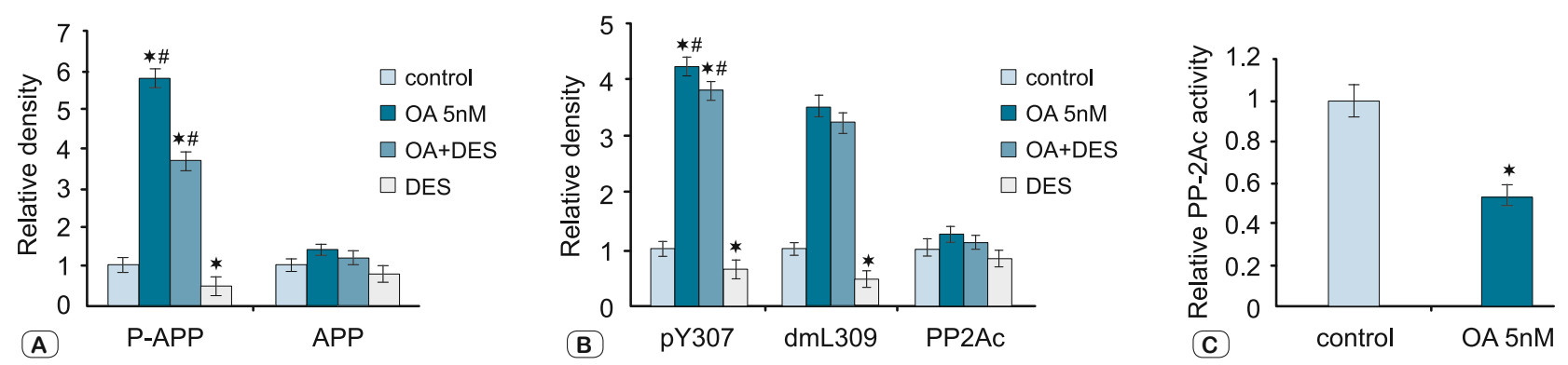

Fig. 2.Effect of Low-concentration OA and DES Specifically Regulating PP-2A Activity on APP Phosphorylation Level. Compared with the control group, * $\mathrm{p}<0.05$; compared with the DES group, $\# \mathrm{p}<0.05$. 
(A)

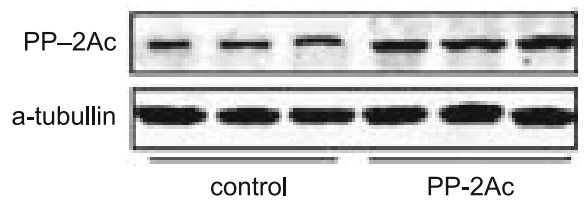

(C)

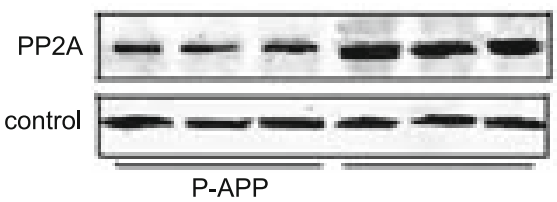

(E)

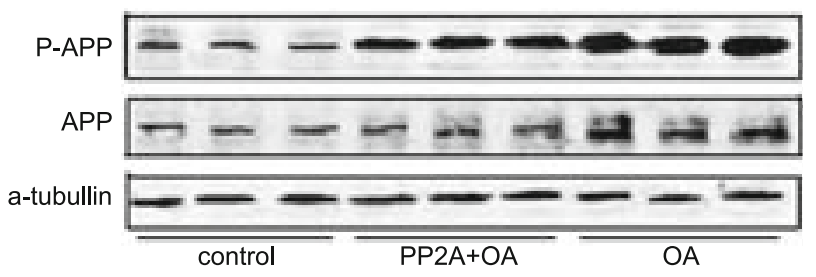

(B)

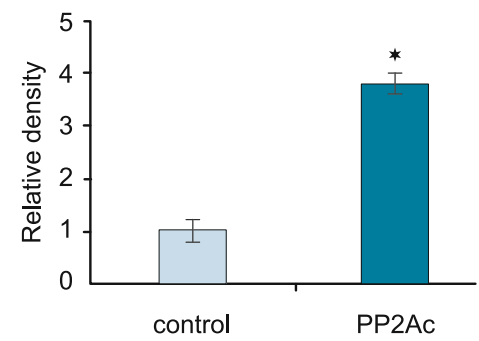

(D)

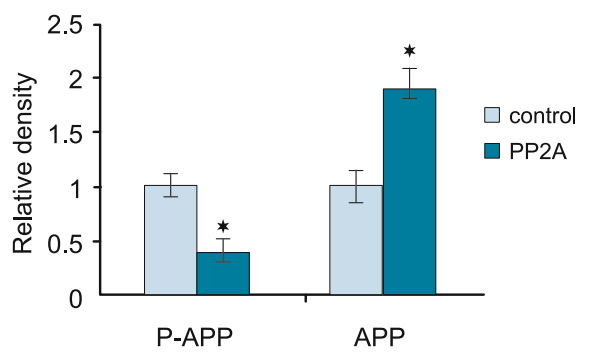

(F)

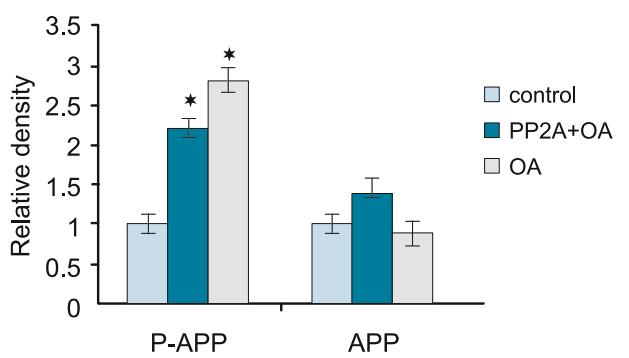

Fig. 3.Over-expressed PP-2A Reducing Phosphorylation Level of APP.The comparison with the control group indicates that * $p<0.05$.

phosphorylation level of the APP668 locus. We have also detected the effect of solely regulating PP1 activity on phosphorylated APP. In the experiment, TM (tautomycin) with concentrations of $5 \mathrm{nM}$ and $25 \mathrm{nM}$ was administered to N2a/APP cells respectively. TM could serve as a specific protein esterase inhibitor and specifically inhibit PP1 activity within the above concentration range. Compared to the control group, the APP phosphorylation level in cells with concentrations of $5 \mathrm{nM}$ and $25 \mathrm{nM}$ exhibited a negative correlation with the dosage of administration indicating that when the PP1 activity was inhibited, the phosphorylation level of the APP668 locus decreased rather than increased (Fig. 1A and 1B). The result excluded the effect of PP1 in rising APP phosphorylation level induced by OA.

Low-concentration $O A$ and DES specifically regulating $P P-2 A$ activity cause corresponding changes in APP phosphorylation

The OA effect dose was $0.1-10 \mathrm{nM}$ and only had inhibitional effect on PP-2A. Therefore, we used $5 \mathrm{nM}$ as the administration concentration to study the role of PP-2A in APP phosphorylation level in the subsequent study. It was observed that the phosphorylation level of APP increased significantly compared to the control group during treating N2a/APP cells with OA for $24 \mathrm{~h}$. PP-2A specificity agonist DES was administered and the phosphorylation level of APP decreased. DES was capable of reversing the rise in APP phosphorylation caused by OA after combined administration
(Fig. 2A). The result has shown that the phosphorylation level of the PP-2AY307 locus of the OA group increased and the demethylation level of L309 decreased after detection of active sites Y307 and L309 of PP-2A. This is exactly converse in the DES group indicating that $\mathrm{OA}$ inhibited PP-2A activity while DES activated PP-2A (Fig. 2B). Meanwhile, we have detected the activity of PP-2A and found that the activity of PP-2A in cells stimulated by $5 \mathrm{nM}$ OA decreased significantly compared to that of the control group (Fig. 2C). The above result has sufficiently demonstrated that PP-2A was able to regulate the phosphorylation level of APP.

Over-expressed PP- $2 A$ can reverse the rise in APP phosphorylation level caused by $O A$

In the previous experiment, we have demonstrated that lowconcentration OA was capable of inhibiting the activity of PP-2A and increasing the phosphorylation level of APP whereas the rise in APP phosphorylation level induced by OA could be reversed after PP-2A was activated by DES. To further verify whether PP2A participated in regulation of the phosphorylation level of APP, we have first over-expressed the PP-2A plasmid in N2a/APP cells (Figs 3A and 3B) and meanwhile tested the expression level of phosphorylated APP. The result has shown that compared to the control group, over-expressed PP-2A significantly decreased the protein level of APP while the total level of APP had no significant changes (Figs 3C and 3D). In addition, we have discovered 
184-190

(A)

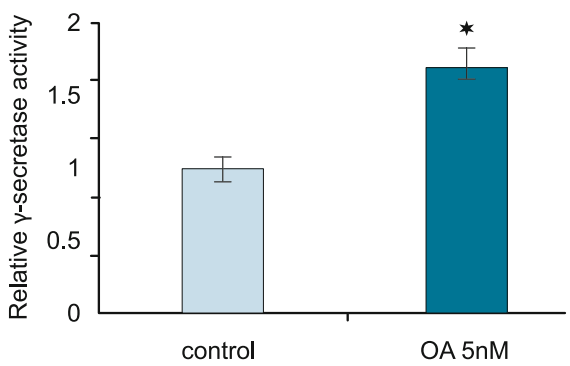

(B)

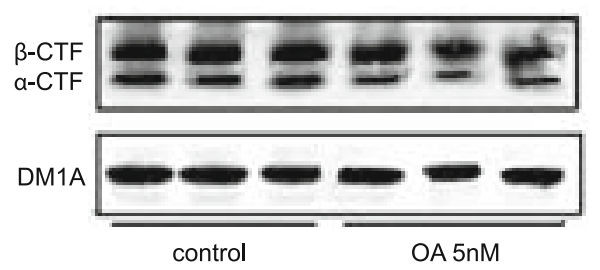

(C)

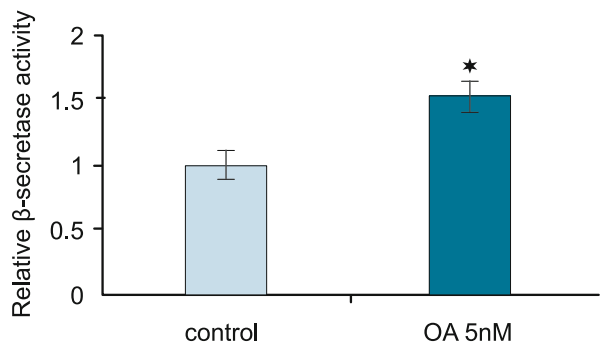

(D)

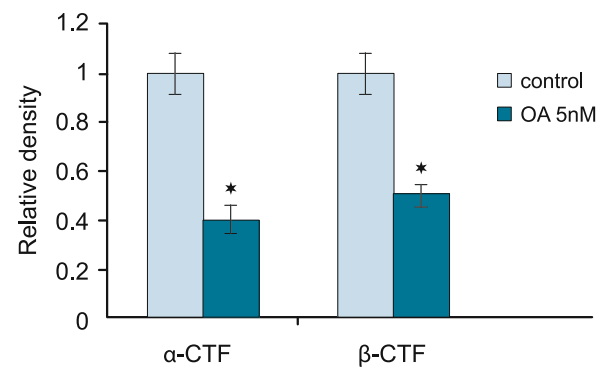

Fig. 4.low-dose $O A$ activating the activities of $\beta$-secretase and $\gamma$-secretase and reducing the contents of $\alpha$-CTF and $\beta$-CTF.The comparison with the control group indicates that $* \mathrm{p}<0.05$.

that the over-expressed PP-2AC was able to reverse the rise in phosphorylation level of APP induced by OA (Figs $3 \mathrm{E}$ and $3 \mathrm{~F}$ ).

Inhibition of PP2A can up-regulate the activity of the $\beta$ and $\gamma$ secretases in N2a/APP cells and reduce output of $\alpha$ and $\beta-C T F$

The protein was extracted with the enzyme activity kit for corresponding enzyme activity detection 24 hours after 5 nM OA stimulation was administered to N2a/APP cells. The result has shown that compared to the control group, the activity of $\beta$-secretase in cells treated with OA increased, the activity of $\gamma$-secretase also increased (Figs 4A and 4B) whereas, the activity of $\alpha$-secretase had no significant changes. The result indicated that the level of APP in cells severed by $\beta$-secretase and $\gamma$-secretase increased accordingly when the phosphorylation level of APP increased. A $\beta$ and $\beta$-CTF form under the action of $\beta$-secretase and $\gamma$-secretase. We have also detected the protein levels of $\alpha-\mathrm{CTF}$ and $\beta-\mathrm{CTF}$ and the result has shown that the content of $\alpha-\mathrm{CTF}$ and $\beta-\mathrm{CTF}$ decreased compared to that of the control group (Figs 4C and 4D).

(A)

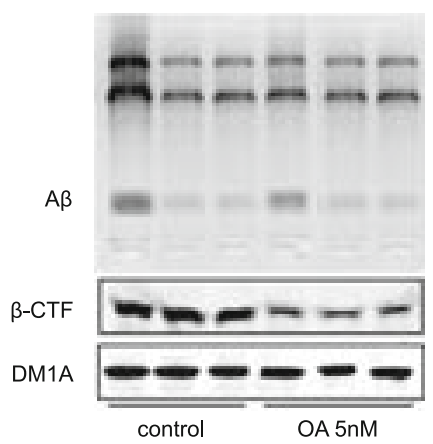

Inhibition of $P P 2 A$ causes rise in $A \beta$ generated

$5 \mathrm{nM}$ low-concentration OA stimulation was administered to the N2a/APP cells. The sample was collected after 24 hours and subjected to western blotting test by gradient tricine gel electrophoresis. The result has shown that compared to the control group $\mathrm{A} \beta$ of OA-treated cells significantly increased indicating that decrease of the PP-2A activity can increase the quantity of A $\beta$ generated by increasing the phosphorylation level of the APP668 site. Meanwhile, we have also observed the protein level of $\beta$-CTF. The protein level of $\beta$-CTF in cells treated by OA significantly decreased compared to that of the control group, which was consistent with the above result (Figure 5A and B).

\section{Discussion}

For Alzheimer's disease, senile plaques caused by amyloid proteinosis in the patient's brain are one of the major pathologic changes. The major constituent is $A \beta$ polypeptide formed from the
(B)

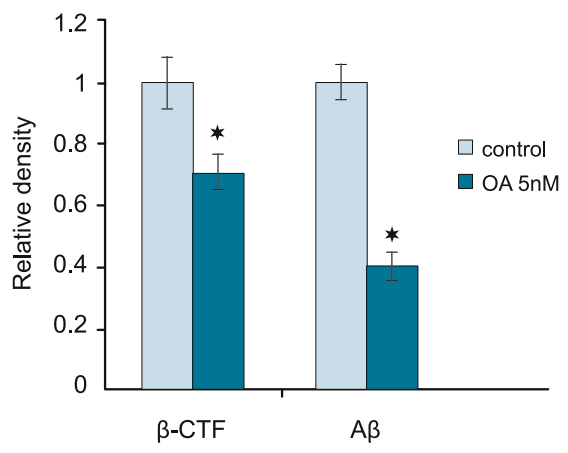

Fig. 5. Low-Dose OA Reducing the Quantity of A $\beta$ Generated. The comparison with the control group indicates that * $p<0.05$. 
amyloid precursor proteosomeAPP severed by secretase(11-12). APP is a transmembrane protein. Its intracellular domain contains multiple phosphorylation sites and two functional domains that can be bound with other proteins (13). Previous studies have shown that phosphorylation of APP threonine 668 site could influence the generation of intracellular $\mathrm{A} \beta$ and high phosphorylation of APP threonine 668 site could strengthen $\mathrm{A} \beta$ generation (14). PP-2A is a protein phosphatase widely present and conservative in cells, which can catalyze in vivo dephosphorylation of the phosphorylated site of the substrate (15). A number of reports have demonstrated that the activity of PP-2A in AD patients was decreasing (16).

APP is a membrane protein, which has a very long N-terminal extracellular fragment (NTF) and a very short C-terminal intracellular fragment (CTF). We have known very well that $\mathrm{C}$-terminal intracellular fragment could be phosphorylated by multiple phosphokinases and the sites (17). It is considered that two elements in the APP intracellular domain could specifically regulate APP, could be severed by secretases, engulfed by cells or A $\beta$ generation. (18). APP phosphorylation can regulate synapse extension in the differentiated PC12 cells (19). The NMR research have indicated that threonine 668 phosphorylation could significantly change APP structurally and maightinfluence binding of APP and other proteins. It is thus considered that threonine 668 phosphorylation could regulate APP intracellular transfer and metabolism (20). Rise in phosphorylation of the threonine 668 site could strengthen the role of secretase in severing amyloid protein generated. Some reports have demonstrated that colocalization existed between APP (P-APP) and BACE1 of the phosphorylated threonine 668 within the hippocampal neurons and primarily cultured cortical neurons of $\mathrm{AD}$ patients and it has been clinically indicated that APP phosphorylation level increased significantly in AD patients' brains (21).

Protein phosphatase $2 \mathrm{~A}$ is a ubiquitous and highly conserved serine/threonine protein esterase, which can be extensively bound with different substrates (22). In many reports, it was stated that imbalance of protein kinase and esterase effects was one of the major factors for $\mathrm{AD}$ onset (23). Research has found that the activity of PP-2A was increased after specifically down-regulating PP-2A specificity inhibitory proteins I2PP-2A in lentivirus infected hAPP transgenic mice and the $A \beta$ level in the brains of the transgenic mice after down-regulating the APP phosphorylation level (24). These research results have indicated that PP-2A maight regulate the phosphorylation level of APP threonine 668 site as an important threonine substrate (25).

PP-2A can reduce the phosphorylation level of APP and it was reported that the rise of the phosphorylation level of APP could increase the $A \beta$ generated (26). Up to now, people still have not been well aware of the pathogenesis of AD but the majority of the scholars have stated that $A \beta$ deposition was the common approach for all factors causing $\mathrm{AD}$ (27). An experiment has shown that $\mathrm{A} \beta$ has neurotoxicity, which can cause neuronal necrosis, apoptosis and amyloidosis (28). It has been generally accepted that the neurotoxicity of $\mathrm{A} \beta$ was the critical factor for $\mathrm{AD}$ formation and progression (29). A $\beta$ accumulation is an early certain event during the $\mathrm{AD}$ onset. As for the onset sequence, $\mathrm{A} \beta$ occurs earlier than pathological changes and clinical symptoms such as nerve fibre entanglement, neuraxon lacking nutrition etc. An experiment has demonstrated that $\mathrm{A} \beta$ was toxic to the cultured nerve cells and also the trophism and toxic effect of $A \beta$ depended on the aggregation state transforming from solubility to insolubility, i.e. $A \beta$ in the aggregation state has a toxic effect (30).

To discuss the effect of changes in the APP phosphorylation level on $A \beta$ generation in our experimental system we detected the changes in quantity of $A \beta$ generated by OA treatment in N2a/APP cells. Small molecular weight protein electrophoresis immunoblotting was used. We have discovered that the $\mathrm{A} \beta$ polypeptide content increased significantly in cells treated by OA. We have detected the activities of $\alpha$-, $\beta$ - and $\gamma$-cand severing products $\beta$-CTF and $\alpha$-CTF due to the fact that the major approach for $\mathrm{A} \beta$ generation was secretase severing. The result has shown that the activities of $\beta$ - and $\gamma$ - secretases increased somewhat whereas the activity of $\alpha$-secretase had no significant change compared to that of the control group. Contents of $\beta$-CTF and $\alpha$-CTF decreased significantly. These results have indicated that the decreased PP-2A activity caused by OA could be increased by raising the APP phosphorylation level, inducing the rise of the activities of $\beta$ - and $\gamma$ - secretases, thus strengthening APP severing. We have discovered that the activities of $\gamma$-secretase and $\beta$-secretase increase, $A \beta$ generated increases and $\beta$-CTF content decreases. The reason may be that the activity of $\gamma$-secretase increasing and acting upon the severed product $\beta$-CTF of $\beta$-secretase produced more $A \beta$ polypeptide and decreased the contents of $\alpha$-CTF and $\beta$-CTF.

Based on the above experimental results, in the research, we have discovered that PP-2A had a direct effect on the APP threonine 668 site in the N2a/APP (stably transfected human APP695) cells. Over-expressed or activating PP-2A by medication decreased the phosphorylation level of the APP668 site and A $\beta$ generated. The phosphorylation level of APP668 site could be raised and the generated $\mathrm{A} \beta$ increased after the PP-2A activity was inhibited by medication. We have also obtained a consistent result in the animals. Meanwhile, we have also discovered that PP-2A was able to influence the activity of APP severing-related secretases and thus PP-2A could regulate secretion of A $\beta$ by influencing activities of phosphorylated $\beta$ - and $\gamma$-secretases at the threonine 668 site.

\section{References}

1. YoumansKL, Tai LMet al. APOE4-specific changes in Abeta accumulation in a new transgenic mouse model of Alzheimer disease. J BiolChem2012; 287 (50): 4174-4186.

2. Tai LMBilousova Tet al. Levels of soluble apolipoprotein E/amyloidbeta(Abeta) complex are reduced and oligomericAbeta increased with APOE4 and Alzheimer disease in a transgenic mouse model and human samples. J Biol Chem2013; 288 (8): 5914-5926.

3. Dore V, Villemagne VLet al.Cross-sectional and Longitudinal Analysis of the Relationship betweenAbeta Deposition, Cortical Thickness, and Memory in Cognitively Unimpaired Individuals and in Alzheimer Disease. JAMA Neurol2013; 70 (7): 903-911.

4. Murakami K, ShimizuT.Cytoplasmic superoxide radical: a possible contributing factor to intracellular Abetaoligomerization in Alzheimer disease. CommunIntegrBiol2012; 5 (3): 255-258. 
184-190

5. TabiraT.Immunotherapy targeting on Abeta for Alzheimer disease. Nihon Rinsho2011; 69 (10): 77-82.

6. RealeM, Kamal MAet al. Relationship between inflammatory mediators, Abeta levels and ApoE genotype in Alzheimer disease. Curr Alzheimer Res2012; 94 (4): 447-457.

7. Butterfield DA, Sultana R.Methionine-35 of abeta (1-42): importance for oxidative stress in Alzheimer disease. J Amino Acids2011; 34 (12): 430-434.

8. Miners JS, Barua Net al.Abeta-degrading enzymes: potential for treatment of Alzheimer disease. J NeuropatholExpNeurol2011; 70 (11): 944-959.

9. Malpass K. Alzheimer disease: Plaque-specific anti-Abeta antibody shows promise in model of AD. Nat Rev Neurol2013; 9 (2): 61-64.

10. McKoyAF, Chen Jet al. A novel inhibitor of amyloid beta(Abeta) peptide aggregation: from high throughput screening to efficacy in an animal model of Alzheimer disease. J BiolChem2012; 287 (46): 38992-38997.

11. FunkeSA, Liu Het al. Identification and characterization of an abeta oligomer precipitating peptide that may be useful to explore gene therapeutic approaches to Alzheimer disease. Rejuvenation Res2012; 15 (2): $144-7$.

12. OzerlatI.Alzheimer disease: CSF biomarkers could be used in Abeta immunotherapy trials for AD. Nat Rev Neurol2012; 8 (6): 297-301.

13. Sato N, Morishita Ret al. Plasma abeta: a possible missing link between Alzheimer disease and diabetes. Diabetes2013; 62 (4): 1005-1006.

14. Uchida Y, Gomi Fet al. Calsyntenin-3 C-terminal fragment accumulates in dystrophic neurites surrounding abeta plaques in tg2576 mouse and Alzheimer disease brains: its neurotoxic role in mediating dystrophic neurite formation. Am J Pathol2013; 82 (5): 1718-1726.

15. Irwin DJ, McMillan CTet al. Comparison of cerebrospinal fluid levels of tau and Abeta 1-42 in Alzheimer disease and frontotemporal degeneration using 2 analytical platforms. Arch Neurol2012; 69 (8): 1018-1025.

16. Ryan TM, FriedhuberAet al. Small amphipathic molecules modulate secondary structure and amyloid fibril-forming kinetics of Alzheimer disease peptide Abeta (1-42). J BiolChem2012; 287 (20): 16947-16954.

17. Alonso E, Vale Cet al. The cholinergic antagonist gymnodimine improves Abeta and tau neuropathology in an in vitro model of Alzheimer disease. Cell PhysiolBiochem2011; 27 (6): 783-794.

18. ChetelatG.Alzheimer disease: Abeta-independent processes-rethinking preclinical AD. Nat Rev Neurol2013; 9 (3): 123-4.
19. WittnamJL, Portelius Eet al.Pyroglutamate amyloid beta(Abeta) aggravates behavioral deficits in transgenic amyloid mouse model for Alzheimer disease. J BiolChem2012; 287 (11): 8154-8162.

20. TengE, Kepe Vet al.(F-18)FDDNP microPET imaging correlates with brain Abeta burden in a transgenic rat model of Alzheimer disease: effects of aging, in vivo blockade, and anti-Abeta antibody treatment. Neurobiol Dis2011; 43 (3): 565-575.

21. VillemagneVL, Pike KEet al. Longitudinal assessment of Abeta and cognition in aging and Alzheimer disease. Ann Neurol2011; 69 (1): 181-192.

22. Butterfield DA. Atorvastatin and Abeta (1-40): not as simple as cholesterol reduction in brain and relevance to Alzheimer disease. ExpNeurol2011; 288 (1): 15-18.

23. CaccamoA, MagriAet al. Age-dependent changes in TDP-43 levels in a mouse model of Alzheimer disease are linked to Abeta oligomers accumulation. MolNeurodegener2010; 5 (1): 51-53.

24. Owen JB, Sultana Ret al. Oxidative modification to LDL receptorrelated protein 1 in hippocampus from subjects with Alzheimer disease: implications for Abeta accumulation in AD brain. Free RadicBiol Med2010; 49 (11): 1798-1803.

25. AluiseCD, Robinson RAet al. Preclinical Alzheimer disease: brain oxidative stress, Abeta peptide and proteomics. Neurobiol Dis2010; 39 (2): $221-228$.

26. PorteliusE, Dean RAet al. A novel Abeta isoform pattern in CSF reflects gamma-secretase inhibition in Alzheimer disease. Alzheimers Res Ther2010; 2 (2): 7-10.

27. Noguchi A, Matsumura Set al. Isolation and characterization of patient-derived, toxic, high mass amyloid beta-protein(Abeta) assembly from Alzheimer disease brains. J BiolChem2009; 284 (47): 32895-32905.

28. Abdullah L, Luis Cet al. Serum Abeta levels as predictors of conversion to mild cognitive impairment/Alzheimer disease in an ADAPT subcohort. Mol Med2009; 15 (11): 432-437.

29. Pavlov PF, Hansson Petersen Cet al. Mitochondrial accumulation of APP and Abeta: significance for Alzheimer disease pathogenesis. J Cell Mol Med2009; 13 (10): 4137-4145.

30. KaminskyYG, Marlatt MWet al. Subcellular and metabolic examination of amyloid-beta peptides in Alzheimer disease pathogenesis: evidence for Abeta (25-35). ExpNeurol2010; 221 (1): 26-37.

Received September 5, 2013. Accepted May 16, 2014. 